International Journal of Pure and Applied Mathematics

Volume 112 No. $2 \quad 2017,341-348$

ISSN: 1311-8080 (printed version); ISSN: 1314-3395 (on-line version)

url: http://www.ijpam.eu

doi: 10.12732/ijpam.v112i2.11

\title{
RADIUS OF STARLIKENESS OF CERTAIN CLASS OF CLOSE-TO-CONVEX FUNCTIONS
}

\author{
Abdullah Yahya ${ }^{1}$, Shaharuddin C. Soh ${ }^{2}$ \\ ${ }^{1}$ Department of Mathematics \\ Faculty of Computers and Mathematical Sciences \\ Universiti Teknologi MARA Cawangan Negeri Sembilan \\ Kampus Seremban, 70300 Seremban, MALAYSIA \\ ${ }^{2}$ Department of Mathematics \\ Faculty of Computers and Mathematical Sciences \\ Universiti Teknologi MARA Malaysia \\ 40450 Shah Alam, Selangor, MALAYSIA
}

\footnotetext{
Abstract: Let $G(\alpha, \delta)$ be the class of normalized analytic functions by $f(0)=f^{\prime}(0)-1=0$ and defined in the open unit disc, $E=\{z:|z|<1\}$ satisfying

$$
\operatorname{Re}\left\{e^{i \alpha} \frac{2 z f^{\prime}(z)}{f(z)-f(-z)}\right\}>\delta,
$$

for $|\alpha|<\pi, \cos \alpha-\delta>0$ and $0 \leq \delta<1$. In this paper, we have determined the bound for $\frac{f(z)}{z}$ and have obtained result for radius of starlikeness, $R_{S t}$ of $G(\alpha, \delta)$.

AMS Subject Classification: 30C45

Key Words: analytic function, bounds, radius of starlikeness
}

\section{Introduction}

Let $\mathcal{A}$ be the class of functions given by

$$
f(z)=z+a_{2} z^{2}+\cdots+a_{n} z^{n}+\cdots=z+\sum_{n=2}^{\infty} a_{n} z^{n}
$$

Received: September 15, 2016

Revised: $\quad$ November 3, 2016

Published: $\quad$ February 1, 2017

$\S_{\text {Correspondence author }}$ (c) 2017 Academic Publications, Ltd. url: www.acadpubl.eu 
which are analytic in $E=\{z:|z|<1\}$. A function $f$ analytic in $E$ is said to be univalent in a domain $D$ if for all $z_{1}, z_{2} \in D, f\left(z_{1}\right)=f\left(z_{2}\right)$ implies $z_{1}=z_{2}$ [2]. The class of all univalent $f$ in $E$ and have a form (1) will be denoted by $S$. We defined subclasses of $S$ consisting of functions that are starlike, convex and close-to-convex denoted by $S t, K$ and $C$ respectively.

Given $f \in A$ and satifying $\left|a_{n}\right| \leq n$ for $n \geq 2$ in $E$, Gavrilov [1] showed that the radius of univalent of the function is real root $r_{0} \simeq 0.164$ in the interval $(0,1)$ of the equation $2(1-r)^{3}-(1+r)=0$ and the result is sharp for $f(z)=2 z-\frac{z}{(1-z)^{2}}$. Consequently, Yamashita [13] showed that the radius of univalence obtained by [1] is also the same as the radius of starlikeness for the function $f \in A$ satisfying $\left|a_{n}\right| \leq n$ for $n \geq 2$. In addition, the author also determined the radius of convexity for the functions $f \in A$ satisfying $\left|a_{n}\right| \leq n$ for $n \geq 2$ is the real root $r_{0} \simeq 0.090$ of the equation $2(1-r)^{4}-\left(1+4 r+r^{2}\right)=0$, while the radius of convexity for functions $f \in A$ satifying $\left|a_{n}\right| \leq M$ for $n \geq 2$ is the real root of $(M+1)(1-r)^{3}-M(1+r)=0$

Kim and Nunokawa [4] investigated some inverse properties for the function $f(z)$ belonging to the class $S$ and satisfying $\left|a_{n}\right| \leq n$ for $n \geq 2$. The authors have proved that $f(z)$ univalent in $|z|<r_{0}$, then $r_{0}$ is the unique solution of the equation $2 r^{3}-6 r^{2}+7 r-1=0$. Nunokawa and Owa [5] have extended results of [4] by showing that the radius of satrlikeness, $R_{S t}>0.08998 \ldots$. Besides that, Ikeda and Saigo [3] showed that the radius of univalent and the radius of convexity by using the Poisson formula satisfying $\left|a_{n}\right| \leq \frac{2}{n},\left|a_{n}\right| \leq$ en and $\left|a_{n}\right| \leq n$ for all $n=2,3, \ldots$. Recently, Yamakawa [12] showed that the radius of starlikeness, $R_{S t}>0.137788 \ldots$ and the radius of convexity, $R_{K}>$ $0.0075450 \ldots$

In this paper, we have defined $G(\alpha, \delta)$ as the class of normalized function $f \in \mathcal{A}$ satisfying the condition

$$
\operatorname{Re}\left\{e^{i \alpha} \frac{2 z f^{\prime}(z)}{f(z)-f(-z)}\right\}>\delta, \quad(z \in E)
$$

where $|\alpha|<\pi, \cos \alpha-\delta>0$ and $0 \leq \delta<1$. These functions are called starlike with respect to symmetric points and were introduced by Sakaguchi [6]. Yahya et al., [8] introduced the generalization of (2) and have showed the coefficient bound, $\left|a_{n}\right| \leq \frac{1}{n}\left[1+(n-1) A_{\alpha, \delta}\right]$ for $n \geq 2$. In addition, the authors also found others extremal properties in $[7,10,11]$ and second Hankel determinant in [9]. Even though there are intensive study on this class of functions, but yet concentrete on the radius properties. Therefore, our purpose on this paper is to determine bound for $\frac{f(z)}{z}$ and obtain result for the radius of starlikeness, $R_{S t}$ of $G(\alpha, \delta)$. 


\section{Preliminary Results}

Based on Yahya et al.,[8], we now find bound for $\frac{f(z)}{z}$ satisfying $\left|a_{n}\right| \leq \frac{1}{n}[1+$ $\left.(n-1) A_{\alpha \delta}\right]$ for $n \geq 2$.

Theorem 1. Let $f \in S$ and $f \in G(\alpha, \delta)$, then

$$
2-\frac{A_{\alpha \delta}}{1-r}+\left(\frac{1-A_{\alpha \delta}}{r}\right) \log (1-r) \leq\left|\frac{f(z)}{z}\right| \leq \frac{A_{\alpha \delta}}{1-r}+\left(\frac{A_{\alpha \delta}-1}{r}\right) \log (1-r)
$$

for $|z|=r<1$. This result is sharp for $\operatorname{Re}\left\{e^{i \alpha} \frac{2 z f^{\prime}(z)}{f(z)-f(-z)}\right\}>\delta$ where $|\alpha|<\pi$, $\cos \alpha-\delta>0$ and $0 \leq \delta<1$.

Proof. Let $\mathcal{A}$ and since $f \in S$ satisfying $\left|a_{n}\right| \leq \frac{1}{n}\left[1+(n-1) A_{\alpha \delta}\right]$ for $n \geq 2$, from (1) we have

$$
|f(z)| \leq|z|+\sum_{n=2}^{\infty}\left|a_{n}\right||z|^{n} .
$$

Based on Yahya et al., [8], $|f(z)|$ is given

$$
|f(z)| \leq|z|+\sum_{n=2}^{\infty}\left(\frac{1}{n}\left[1+(n-1) A_{\alpha \delta}\right]\right)|z|^{n} .
$$

We simplify

$$
|f(z)| \leq|z|+\sum_{n=2}^{\infty} \frac{|z|^{n}}{n}+A_{\alpha \delta} \sum_{n=2}^{\infty}|z|^{n}-A_{\alpha \delta} \sum_{n=2}^{\infty} \frac{|z|^{n}}{n} .
$$

Since $\log (1-|z|)=-\sum_{n=2}^{\infty} \frac{|z|^{n}}{n}$ and $\frac{1}{1-|z|}=\sum_{n=2}^{\infty}|z|^{n}$, thus $|f(z)| \leq|z|+(-|z|-\log (1-|z|))+A_{\alpha \delta}\left(-1-|z|+\frac{1}{1-|z|}\right)-A_{\alpha \delta}(-|z|-\log (1-|z|))$

which gives

$$
\left|\frac{f(z)}{z}\right| \leq \frac{A_{\alpha \delta}}{1-r}+\left(\frac{A_{\alpha \delta}-1}{r}\right) \log (1-r),
$$

for $|z|=r<1$. Therefore, $f(z)$ absolutely converge in $E$, and so, $f(z)$ analytic in $E$. On the other hand, we have

$$
|f(z)| \geq|z|-\sum_{n=2}^{\infty}\left|a_{n}\right||z|^{n}
$$


and

$$
|f(z)| \geq|z|-\sum_{n=2}^{\infty}\left(\frac{1}{n}\left[1+(n-1) A_{\alpha \delta}\right]\right)|z|^{n}
$$

We simplify

$$
|f(z)| \geq|z|-\sum_{n=2}^{\infty} \frac{|z|^{n}}{n}-A_{\alpha \delta} \sum_{n=2}^{\infty}|z|^{n}+A_{\alpha \delta} \sum_{n=2}^{\infty} \frac{|z|^{n}}{n} .
$$

and

$$
\begin{aligned}
|f(z)| & \geq|z|-\left(-|z|+\sum_{n=2}^{\infty} \frac{|z|^{n}}{n}\right) \\
& -A_{\alpha \delta}\left(1-|z|+\sum_{n=2}^{\infty}|z|^{n}\right)+A_{\alpha \delta}\left(1|z|+\sum_{n=2}^{\infty} \frac{|z|^{n}}{n}\right) .
\end{aligned}
$$

So that

$$
|f(z)| \geq 2|z|+\log (1-|z|)+A_{\alpha \delta}-\frac{A_{\alpha \delta}}{1-|z|}-A_{\alpha \delta} \log (1-|z|),
$$

which gives

$$
\left|\frac{f(z)}{z}\right| \geq 2-\frac{A_{\alpha \delta}}{1-r}+\left(\frac{1-A_{\alpha \delta}}{r}\right) \log (1-r),
$$

for $|z|=r<1$ as required. The following corollary is immediate.

Corollary 2. Let $f \in G(\alpha, \delta)$ and for $|z|=r<1$, then

$$
|z|<R_{1}=0.7475842618
$$

for $0 \leq R_{1} \leq r<1$. Thus Theorem 1 is sharp.

Proof. By means of Theorem 1, we have $\left|\frac{f(z)}{z}\right|>0$, thus

$$
\left|\frac{f(z)}{z}\right| \geq 2-\frac{A_{\alpha \delta}}{1-R_{1}}+\left(\frac{1-A_{\alpha \delta}}{R_{1}}\right) \log \left(1-R_{1}\right)=0 .
$$

Based on Yahya et al.,[9], from $A_{\alpha \delta}=\cos \alpha-\delta \leq 0.9433$, we have

$$
2-\frac{0.9433}{1-R_{1}}+\left(\frac{1-0.9433}{R_{1}}\right) \log \left(1-R_{1}\right)=0
$$

and yield $R_{1}=0.7475842618$ as required. 


\section{Main Result}

We now will estimate radius of starlikeness, $R_{S t}$ that satisfying the class $G(\alpha, \delta)$ in $E$. We begin with the following corollary.

Corollary 3. Let $u(z)$ be harmonic in $|z| \leq \rho$ and continuous in $|z| \leq \rho$. Then $u(z)$ is given by the equation

$$
u(z)=u\left(r e^{i \theta}\right)=\frac{1}{2 \pi} \int_{0}^{2 \pi} \Re\left\{u\left(\rho e^{i \phi}\right)\right\} \frac{\rho^{2}-r^{2}}{\rho^{2}-2 \rho r \cos (\phi-\theta)+r^{2}} d \phi,
$$

where $0 \leq r<\rho$.

The (3) is called the Poisson formula.[3]

Theorem 4. Let $f \in \mathcal{A}$ and $f \in G(\alpha, \delta)$, then $f$ is univalent and starlike in $|z|<R_{S t}$, where

$$
R_{S t}=0.2655158289 \text {. }
$$

Proof. By means of Theorem 1, we have $\left|\frac{f(z)}{z}\right|>0$ in $|z|<R_{1}=0.7475842$ 618, and therefore $\log \left(\frac{f(z)}{z}\right)$ is harmonic in $|z|<R_{1}$. From Corollary 3 and based on the harmonic function theory, we defined

$$
\log \frac{f(z)}{z}=\frac{1}{2 \pi} \int_{0}^{2 \pi}\left(\log \left|\frac{f(\zeta)}{\zeta}\right|\right) \frac{\zeta+z}{\zeta-z} d \phi
$$

where $\zeta=\rho e^{i \phi},(0 \leq \phi \leq 2 \pi), z=r e^{i \theta},(0 \leq \theta \leq 2 \pi)$ and $0 \leq r<\rho \leq R_{1}=$ 0.7475842618 .

By using the logarithmic differentiation, we have

$$
\frac{d}{d z}\left(\log \frac{f(z)}{z}\right)=\frac{1}{2 \pi} \int_{0}^{2 \pi}\left(\log \left|\frac{f(\zeta)}{\zeta}\right|\right) \frac{d}{d z}\left(\frac{\zeta+z}{\zeta-z}\right) d \phi
$$

and yield

$$
\frac{z}{f(z)}\left(\frac{f^{\prime}(z)}{z}-\frac{f(z)}{z^{2}}\right)=\frac{1}{2 \pi} \int_{0}^{2 \pi}\left(\log \left|\frac{f(\zeta)}{\zeta}\right|\right)\left(\frac{(\zeta-z)-(\zeta+z)}{(\zeta-z)^{2}}\right) d \phi .
$$

We can simplify (4) as

$$
\frac{1}{z}\left(\frac{z f^{\prime}(z)}{f(z)}-1\right)=\frac{1}{2 \pi} \int_{0}^{2 \pi}\left(\log \left|\frac{f(\zeta)}{\zeta}\right|\right)\left(\frac{2 \zeta}{(\zeta-z)^{2}}\right) d \phi
$$

and

$$
\frac{z f^{\prime}(z)}{f(z)}=1+\frac{1}{2 \pi} \int_{0}^{2 \pi}\left(\log \left|\frac{f(\zeta)}{\zeta}\right|\right)\left(\frac{2 z \zeta}{(\zeta-z)^{2}}\right) d \phi
$$


From (5), we have

$$
\frac{z f^{\prime}(z)}{f(z)}=1+\frac{1}{2 \pi} \int_{0}^{2 \pi}\left(|\log | \frac{f(\zeta)}{\zeta}||\right)\left|\frac{2 z \zeta}{(\zeta-z)^{2}}\right| d \phi
$$

and yield

$$
\frac{z f^{\prime}(z)}{f(z)}=1+\frac{1}{2 \pi} \int_{0}^{2 \pi}\left(|\log | \frac{f(\zeta)}{\zeta}||\right) \frac{2\left|r e^{i \theta}\right|\left|\rho e^{i \phi}\right|}{\left|\rho e^{i \phi}-r e^{i \theta}\right|^{2}} d \phi .
$$

From $\left|\rho e^{i \phi}\right|=\rho$ and $\left|r e^{i \theta}\right|=r$, we have

$\frac{z f^{\prime}(z)}{f(z)}=1+\frac{1}{2 \pi} \int_{0}^{2 \pi}\left(|\log | \frac{f(\zeta)}{\zeta}||\right) \frac{2 r \rho}{|(\rho \cos \phi-r \cos \theta)+i(\rho \sin \phi-r \sin \theta)|^{2}} d \phi$,

which gives

$\frac{z f^{\prime}(z)}{f(z)}=1+\frac{1}{2 \pi} \int_{0}^{2 \pi}\left(|\log | \frac{f(\zeta)}{\zeta}||\right) \frac{2 r \rho}{(\rho \cos \phi-r \cos \theta)^{2}+(\rho \sin \phi-r \sin \theta)^{2}} d \phi$.

So that

$$
\frac{z f^{\prime}(z)}{f(z)}=1+\frac{1}{2 \pi} \int_{0}^{2 \pi}\left(|\log | \frac{f(\zeta)}{\zeta}||\right) \frac{2 r \rho}{\rho^{2}-2 r \rho \cos (\rho-\theta)+r^{2}} d \phi .
$$

From Theorem 1, we have

$$
2-\frac{A_{\alpha \delta}}{1-r}+\left(\frac{1-A_{\alpha \delta}}{r}\right) \log (1-r)<\frac{A_{\alpha \delta}}{1-r}+\left(\frac{A_{\alpha \delta}-1}{r}\right) \log (1-r)
$$

for $|z|=r<1$. We derive

$$
\Re\left\{\frac{z f^{\prime}(z)}{f(z)}\right\} \geq 1-\frac{1}{2 \pi} \int_{0}^{2 \pi} \max _{|\phi|=\rho}\left(|\log | \frac{f(\zeta)}{\zeta}||\right) \frac{2 r \rho}{\rho^{2}-2 r \rho \cos (\rho-\theta)+r^{2}} d \phi
$$

and from (6) and integrating with respect to $\phi$ yield

$$
\Re\left\{\frac{z f^{\prime}(z)}{f(z)}\right\} \geq 1-\frac{2 r \rho}{\rho^{2}-r^{2}} \log \left(\frac{A_{\alpha \delta}}{1-\rho}+\left(\frac{A_{\alpha \delta}-1}{\rho}\right) \log (1-\rho)\right)
$$

where $0 \leq r<\rho \leq R_{1}=0.7475842618$.

Based on $\Re\left\{\frac{z f^{\prime}(z)}{f(z)}\right\}>0$ in $|z|<R_{S t}$ and from (7), we see that

$$
\frac{2 r \rho}{\rho^{2}-r^{2}} \log \left(\frac{A_{\alpha \delta}}{1-\rho}+\left(\frac{A_{\alpha \delta}-1}{\rho}\right) \log (1-\rho)\right)=1
$$


and

$$
\log \left(\frac{A_{\alpha \delta}}{1-\rho}+\left(\frac{A_{\alpha \delta}-1}{\rho}\right) \log (1-\rho)\right)=\frac{\rho^{2}-r^{2}}{2 r \rho}
$$

Substituting $\rho=(1+\sqrt{2}) r$ in $(8)$, we have

$$
\log \left(\frac{A_{\alpha \delta}}{1-(1+\sqrt{2}) r}+\left(\frac{A_{\alpha \delta}-1}{(1+\sqrt{2}) r}\right) \log (1-(1+\sqrt{2}) r)\right)=1
$$

which gives

$$
\frac{A_{\alpha \delta}}{1-(1+\sqrt{2}) r}+\left(\frac{A_{\alpha \delta}-1}{(1+\sqrt{2}) r}\right) \log (1-(1+\sqrt{2}) r)=e,
$$

in $|z|<R_{S t}$. Based on Yahya et al.,[9], from $A_{\alpha \delta}=\cos \alpha-\delta \leq 0.9433$, we have

$$
R_{S t}=0.2655158289
$$

as required.

\section{Acknowledgments}

This research was supported by the Universiti Teknologi MARA (UiTM) under the Geran Dana Pembudayaan Penyelidikan (iRAGS), 600-RMI/DANA 5/3/IRAGS(22/2015). The author(s) would like to acknowledge UiTM and Institut Pengurusan Penyelidikan dan Inovasi (IRMI) for the support and contributions.

\section{References}

[1] V.I. Gavrilov, Remarks on the radius of univalence of holomorphic functions, Matematicheskie Zametki, 7 (1970), 295-298.

[2] A.W. Goodman, Univalent Function Volume 1,Mariner Publishing Co. Inc. Tampa: University of South Florida, (1983).

[3] A. Ikeda and M. Saigo, Various inverse problems for univalent functions, RIMS Kokyu Record, 1164 (2000), 22-30.

[4] Y.C. Kim and M. Nunokawa, On some radius results for certain analytic functions, Kyungpook Mathematical Journal, 37 (1997), 61-65.

[5] M. Nunokawa and S. Owa, On some inverse properties for univalent functions, Kyoto University Koukyuroku, 1164 (2000), 73-76. 
[6] K. Sakaguchi, On a Certain Univalent Mapping, J. Mathematical Society of Japan, 11 (1959).

[7] A. Yahya, S.C. Soh and D. Mohamad, Arc length and Area of certain class of close-toconvex functions, Proceeding of The 10TH IMT-GT International Conference on Mathematics, Statistics and Its Application 2014, 10 (2014), 24-32.

[8] A. Yahya, S.C. Soh and D. Mohamad, Coefficient bound of a generalized close-to-convex function, International Journal of Pure and Applied Mathematics, 83 (2013), 287-293.

[9] A. Yahya, S.C. Soh and D. Mohamad, Second Hankel determinant for a class of a generalised Sakaguchi class of analytic functions, International Journal of Mathematical Analysis, 33 (2013), 1601-1608.

[10] A. Yahya, S.C. Soh and D. Mohamad, Some extremal properties of a generalized close-toconvex functions, International Journal of Mathematical Analysis, 39 (2014), 1931-1936.

[11] A. Yahya, S.C. Soh and D. Mohamad, Some extremal properties of a generalized Sakaguchi class of analytic functions, Proceeding of the 20th National Symposium on Mathematical Sciences, 1522 (2013), 920-925.

[12] R. Yamakawa, Radius of starlikeness and convexity 2, RIMS Kokyu Record, 1363 (2004), 131-135.

[13] S. Yamashita, Radii of univalence, starlike and convexity, Bulletin of the Australian Mathematical Society, 25 (1982), 453-457. 